\title{
Extraordinary Mechanism of the Diels-Alder Reaction: Investigation of Stereochemistry, Charge Transfer, Charge Polarization, and Biradicaloid Formation \\ Supporting Information
}

Thomas M. Sexton, Elfi Kraka, and Dieter Cremer*

Computational and Theoretical Chemistry Group (CATCO), Department of Chemistry, Southern Methodist University, 3215 Daniel Ave, Dallas, Texas 75275-0314, USA E-mail: dcremer@smu.edu 


\section{Contents}

1 Harmonic Frequencies and Avoided Crossings for the Retro Diels-Alder Reaction as a Function of the Reaction Parameter $s$

2 Curvature, Energy, and Geometry Diagrams $\quad$ S6

3 Ball\&Stick Representations of the Reaction Complex at Selected Points Along the Reaction Path

4 Cartesian Coordinates of the Reaction Complex at Selected Points Along the Reaction Path 


\section{Harmonic Frequencies and Avoided Crossings for the Retro Diels-Alder Reaction as a Function of the Reaction Pa- rameter $s$}

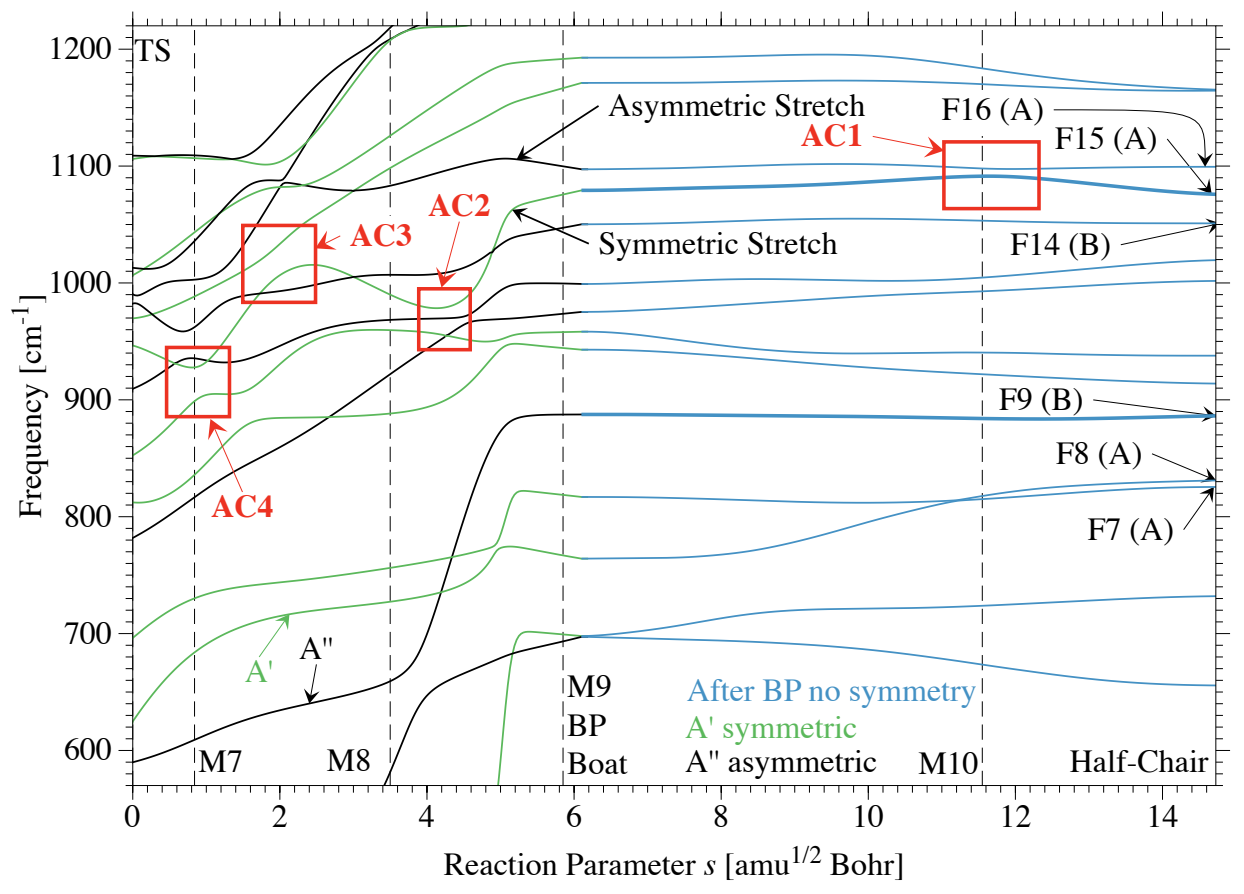

Figure S1: Harmonic vibrational frequencies in the exit channel of the Diels-Alder reaction as a function of the reaction parameter $s$. The zero point of the path parameter defines the location of the TS. Regions of high Coriolis Coupling between modes (Avoid Crossings, AC) are indicated by red boxes. Frequencies corresponding to A'-symmetrical modes are given in green and those corresponding to A"-symmetrical modes in black. The bifurcation point is near $s=-6.1$ units. After the bifurcation point, all vibrational modes are $C_{1}$-symmetrical and the corresponding frequencies are indicated in blue. The half chair has $\mathrm{C}_{2}$ symmetry. The strongest symmetric stretching mode for C1C6/C4C5 is F15. During ring conversion from half chair to boat, this mode undergoes an avoided crossing (AC1) with F16. Between the boat formation and the TS, both modes undergo numerous avoided crossings. The strongest asymmetric stretching mode for $\mathrm{C} 1 \mathrm{C} 6 / \mathrm{C} 4 \mathrm{C} 5$ is F9. During ring conversion, this mode does not undergo any avoided crossings. The reaction path is symmetric, so energy in this mode cannot be transferred to the path for the concerted retro Diels-Alder reaction. 
Table S1: Character of some normal modes Fn of the reaction complex in the exit channel. ${ }^{a}$

\begin{tabular}{|l|l|r|}
\hline Frequency & Symmetry & $\begin{array}{r}\text { C1C6/C4C5 } \\
\text { Contribution }\end{array}$ \\
\hline F16 & A & Negligible \\
F15 & A & $30.1 \%$ \\
F14 & B & $12.9 \%$ \\
F9 & B & $20.2 \%$ \\
F8 & A & $11.1 \%$ \\
F7 & A & $13.1 \%$ \\
\hline
\end{tabular}

${ }^{a}$ Compare with Figure S1. Frequency number Fn, symmetry, and the contribution of the corresponding normal mode to the local C1C6/C4C5 stretching mode of cyclohexene are listed. The normal mode decomposition was carried out using the local mode analysis of Konkoli and Cremer, see manuscript. 


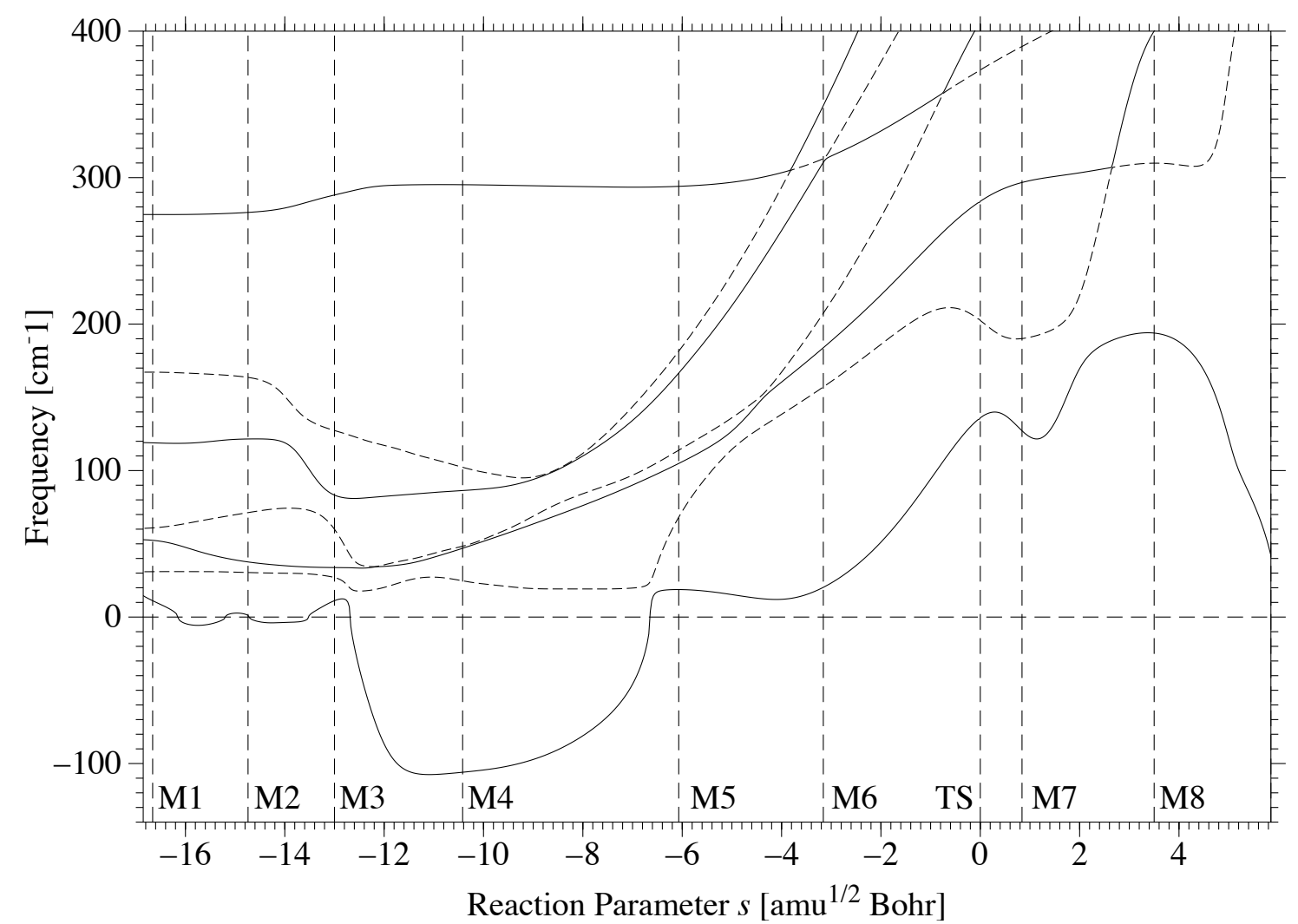

Figure S2: B3LYP/6-31G(d,p). Lowest vibrational frequencies of the reaction complex. A path instability caused by a VRI point at -6.7 s-units and a ridge path out into the entrance channel are indicated by imaginary values of the lowest frequency (indicated by a ngative value). 


\section{Curvature, Energy, and Geometry Diagrams}

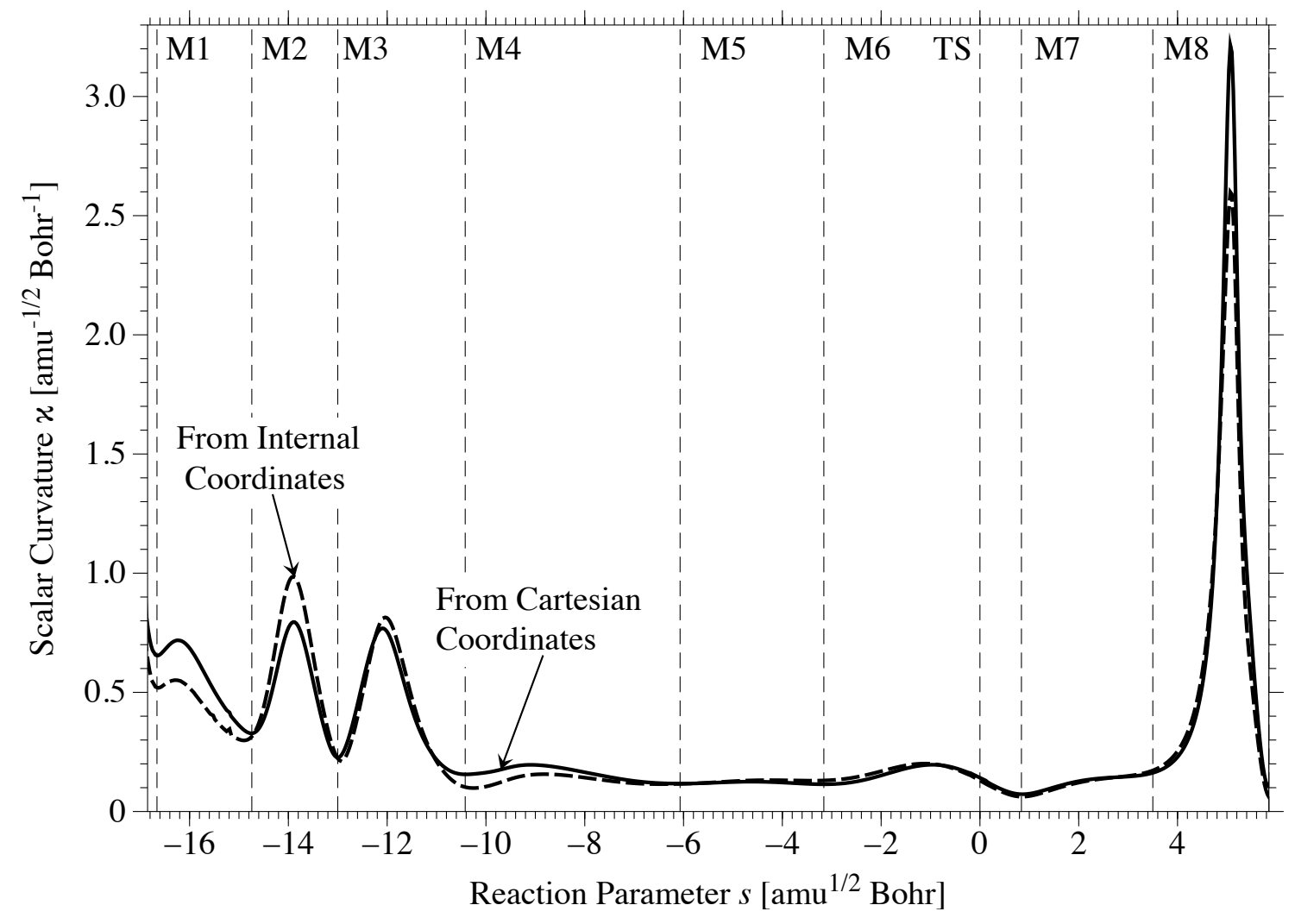

Figure S3: B3LYP/6-31G(d,p). Scalar curvature diagram of the Diels-Alder reaction given in terms of Cartesian coordinates (solid line) and internal coordinates (dashed line). The later deviates from the correct scalar curvature at places where the internal coordinates chosen for the calculation of the scalar curvature do not span the (3N-L)-dimensional space (N: number of atoms; L: number of rotations and translations) correctly. 


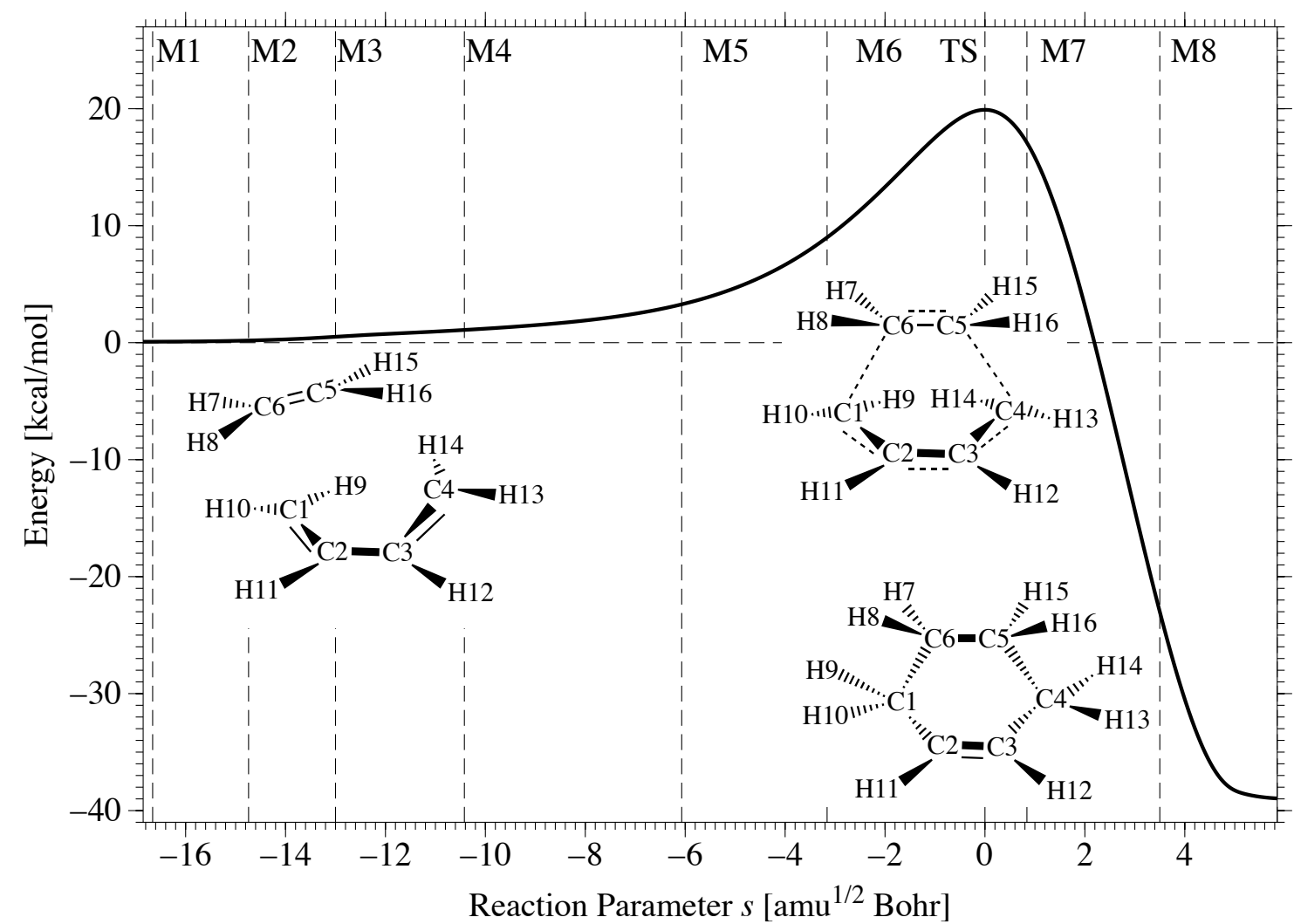

Figure S4: B3LYP/6-31G(d,p). Energy diagram of the Diels-Alder reaction. The exit channel ends at the boat configuration, which is a bifurcation point. The cyclohexene ring can follow two equivalent pseudorotation paths to adopt half chair conformations of cyclohexene, which are related by an inversion. 


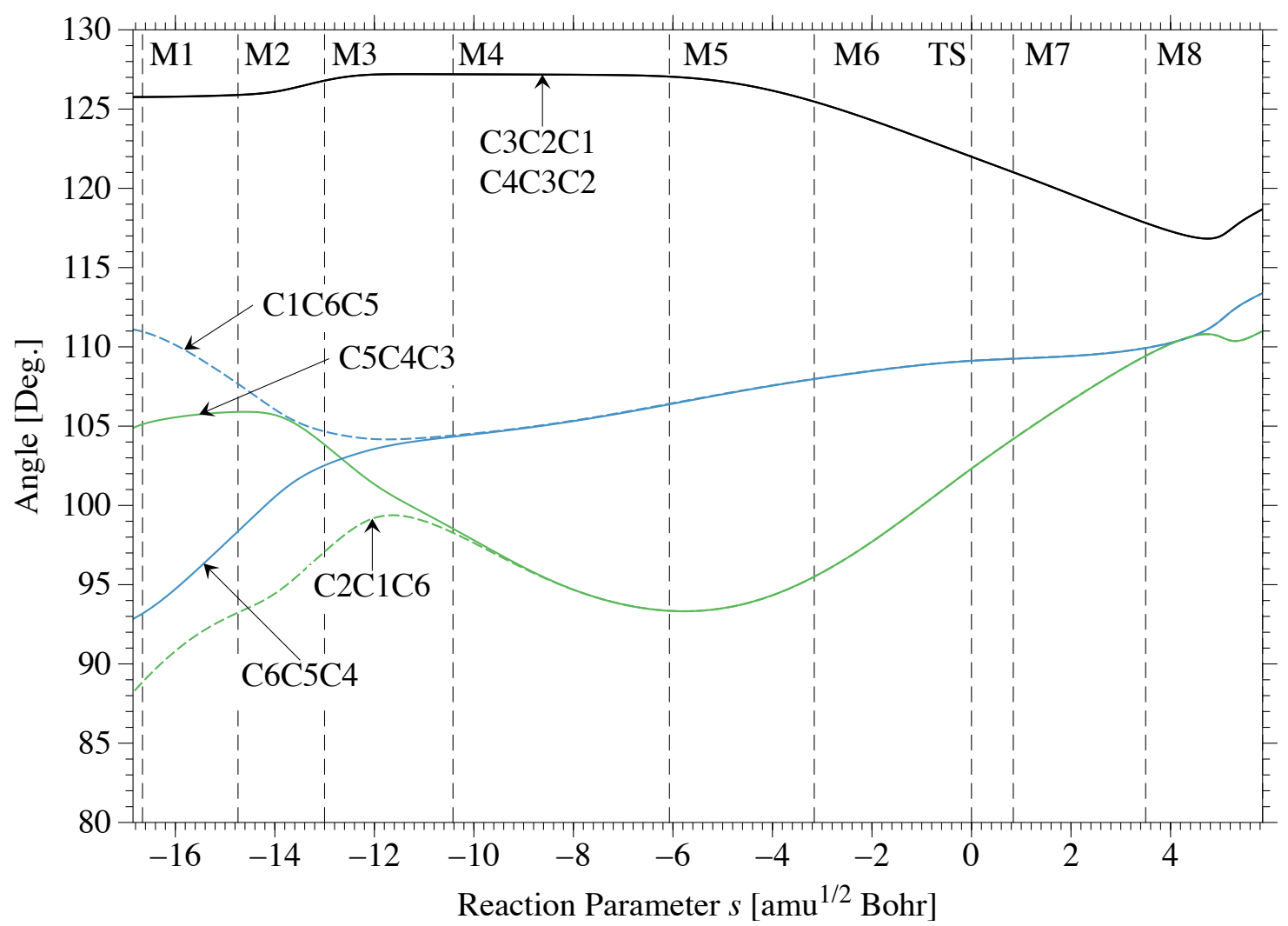

Figure S5: B3LYP/6-31G(d,p). Changes in the bond angles are given as a function of the reaction parameter $s$. 


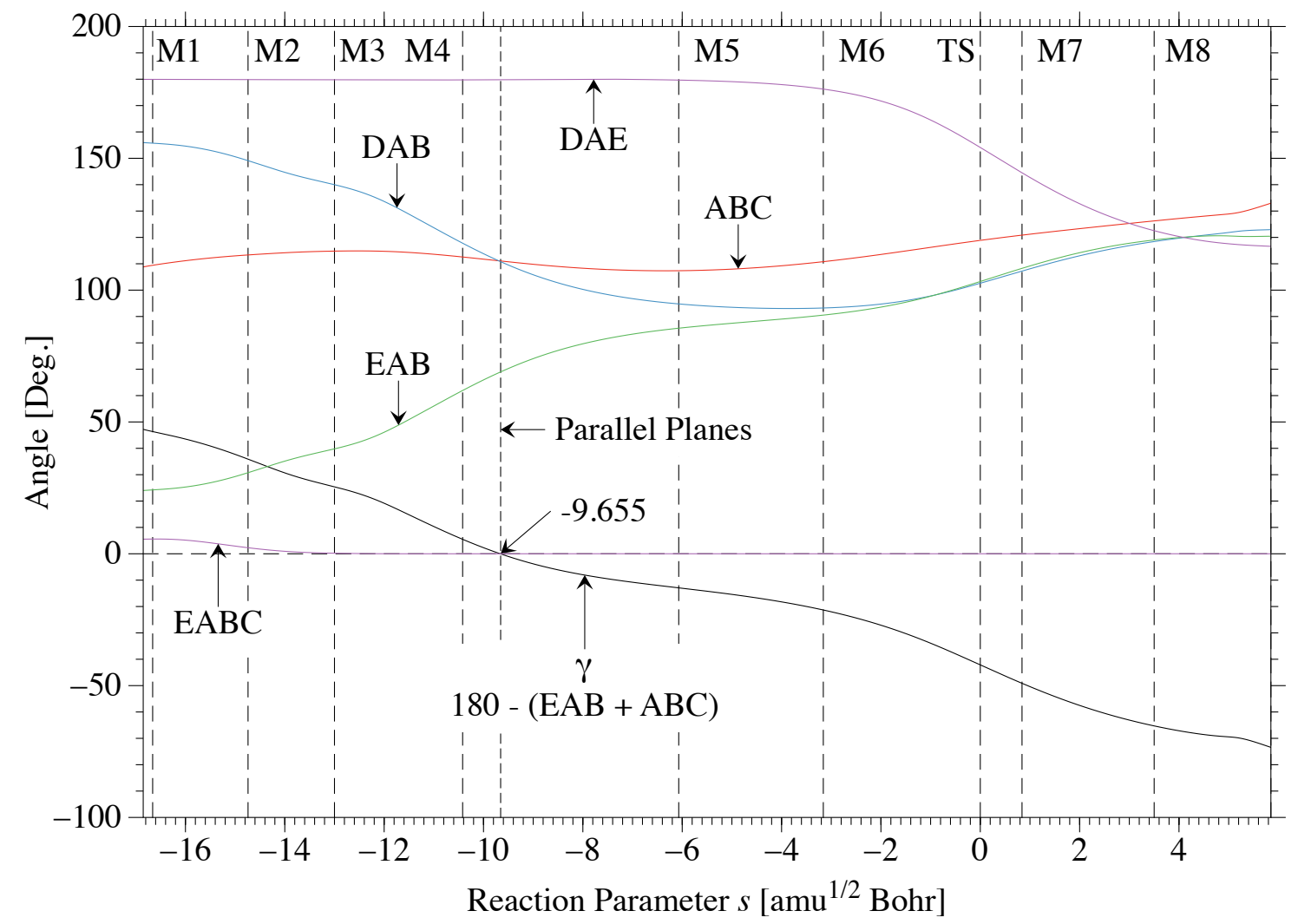

Figure S6: B3LYP/6-31G(d,p). Changes in specific angles (defined by auxiliary points A, B, C, D, E) given as a function of the reaction parameter $s$. See Figure 1 in the manuscript for the definition of the auxiliary points $\mathrm{A}, \mathrm{B}, \mathrm{C}, \mathrm{D}$, and E. 


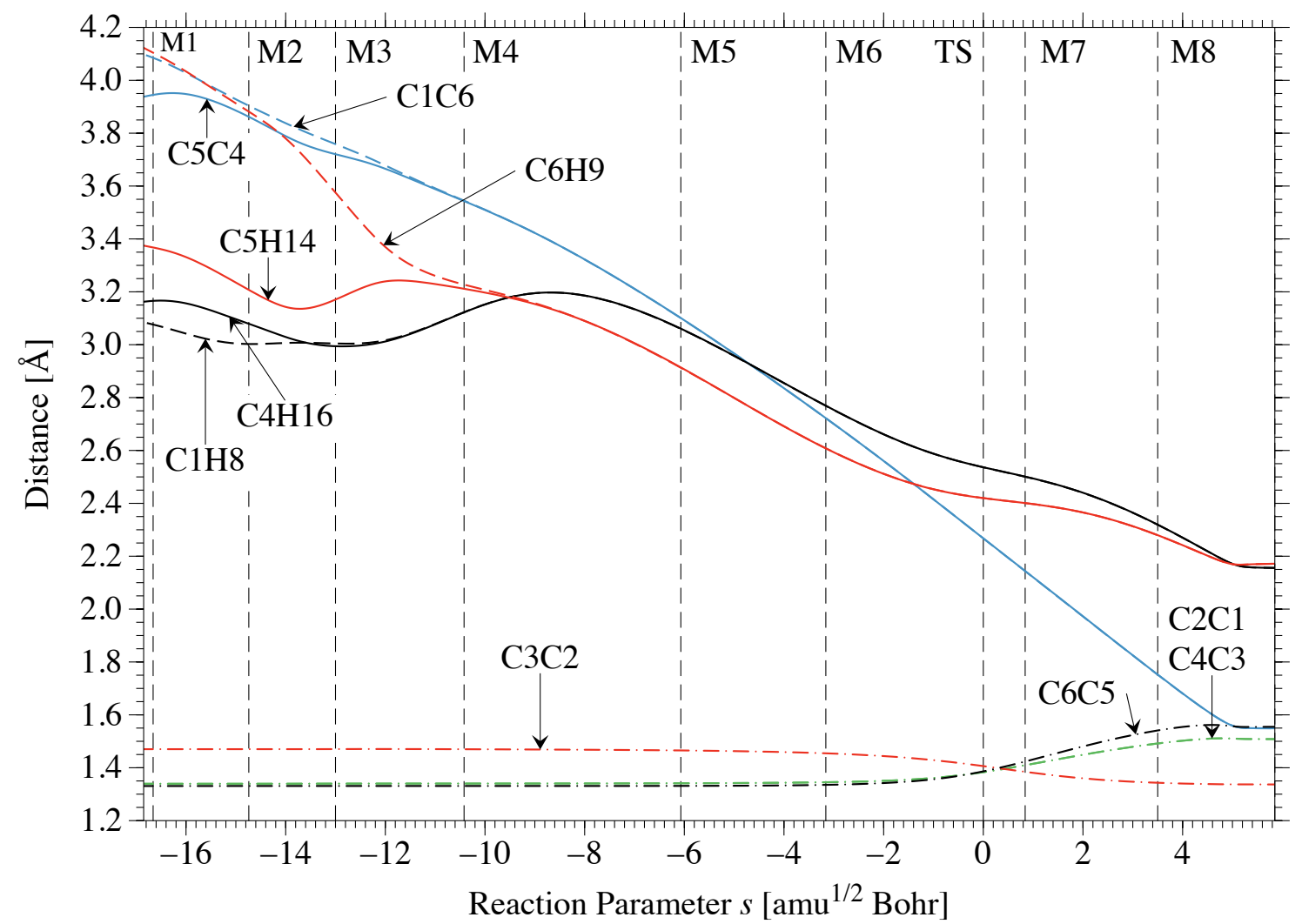

Figure S7: B3LYP/6-31G(d,p). Changes in specific approach distances given as a function of the reaction parameter $s$. After M4, the reaction complex adopts $C_{s}$-symmetry as indicated by the merging of various distance functions. 


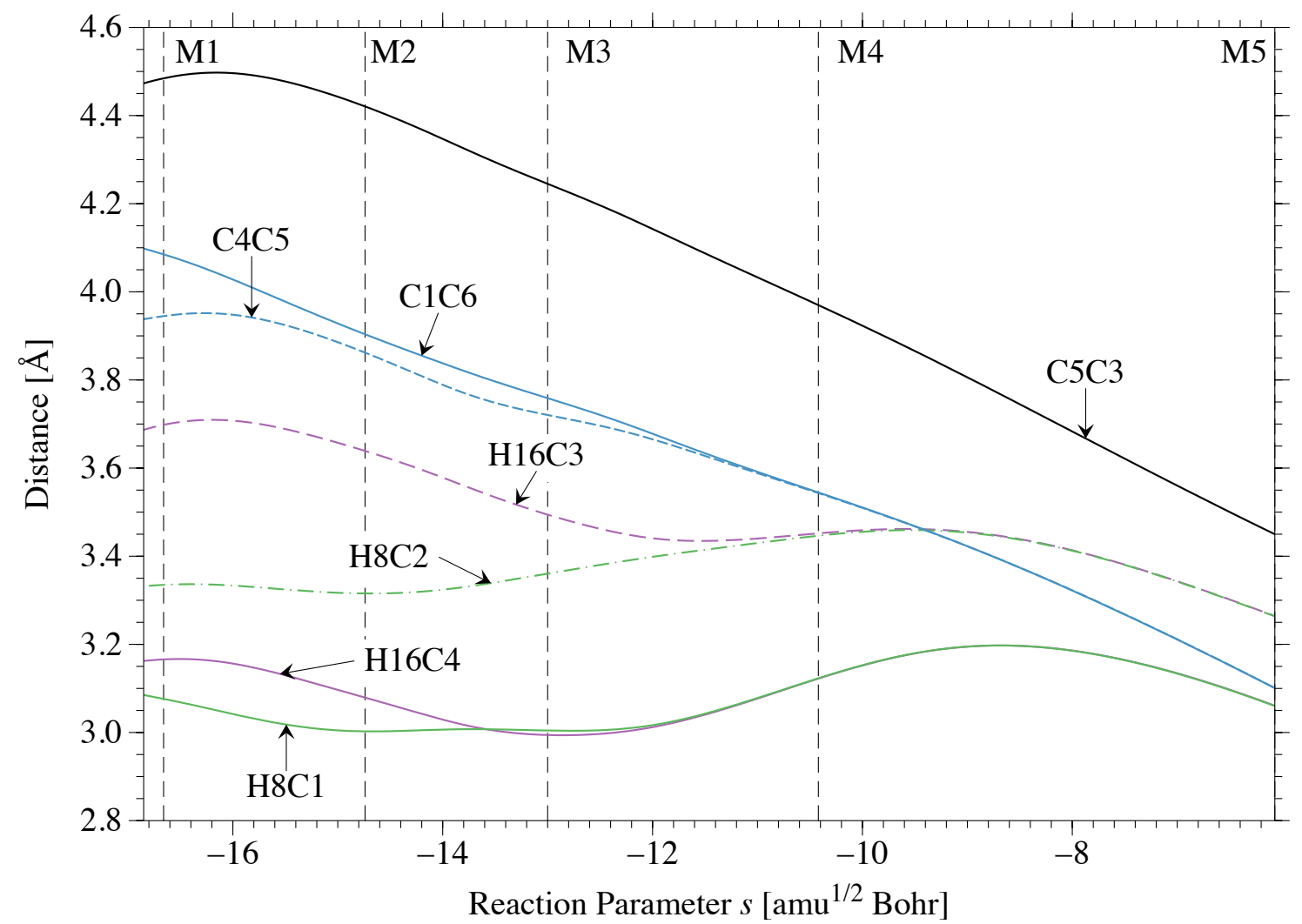

Figure S8: B3LYP/6-31G(d,p). Changes in specific approach distances in the entrance channel given as a function of the reaction parameter $s$. After M4, the reaction complex adopts $C_{s}$-symmetry as indicated by the merging of various distance functions. 


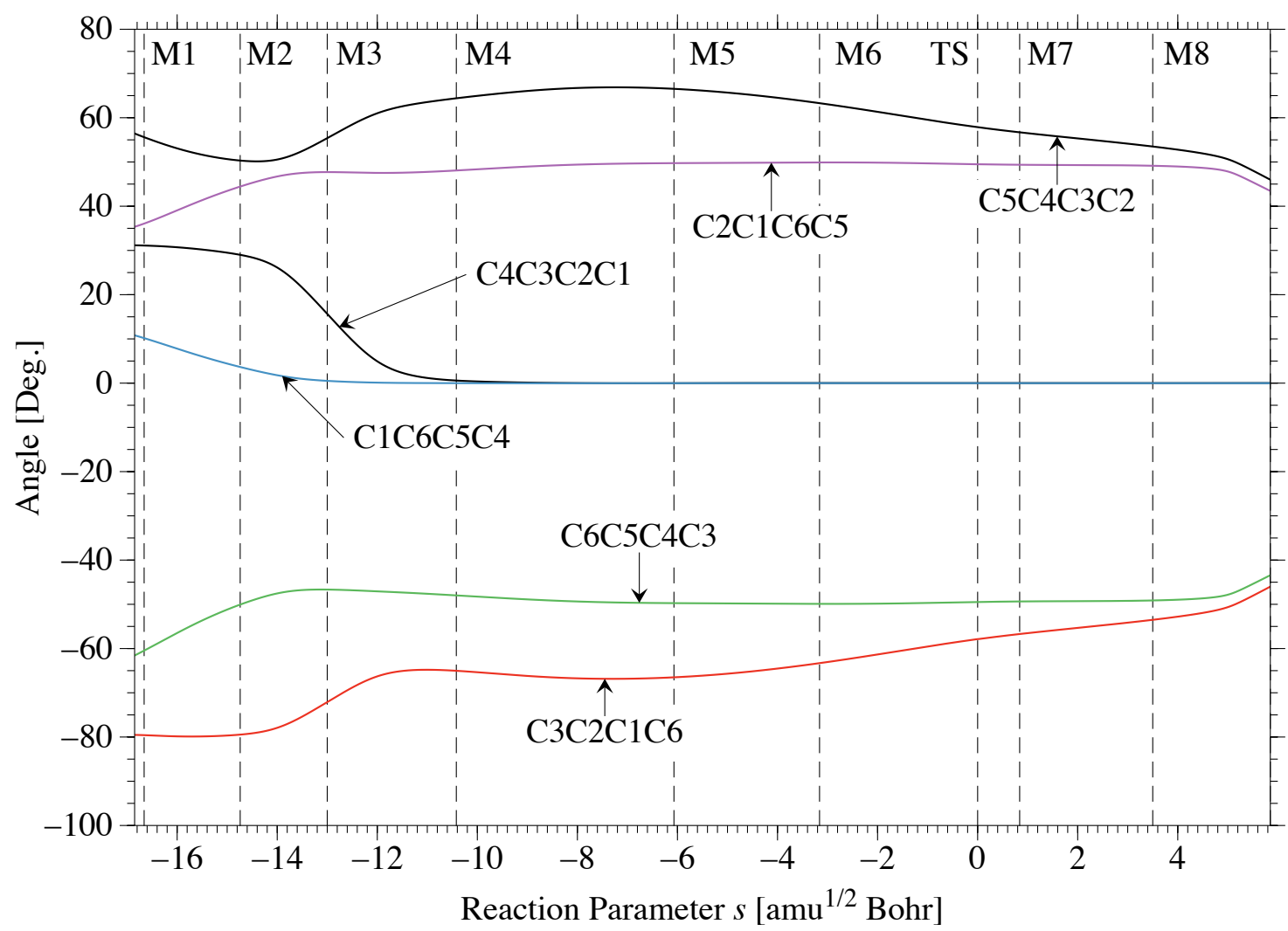

Figure S9: B3LYP/6-31G(d,p). Changes in specific dihedral angles given as a function of the reaction parameter $s$. After $\mathrm{M} 4, \mathrm{C} 4 \mathrm{C} 3 \mathrm{C} 2 \mathrm{C} 1=0$, thus confirming the $C_{s}$-symmetry of the $\mathrm{RC}$ beyond this point. 


\section{Ball\&Stick Representations of the Reaction Complex at Selected Points Along the Reaction Path}

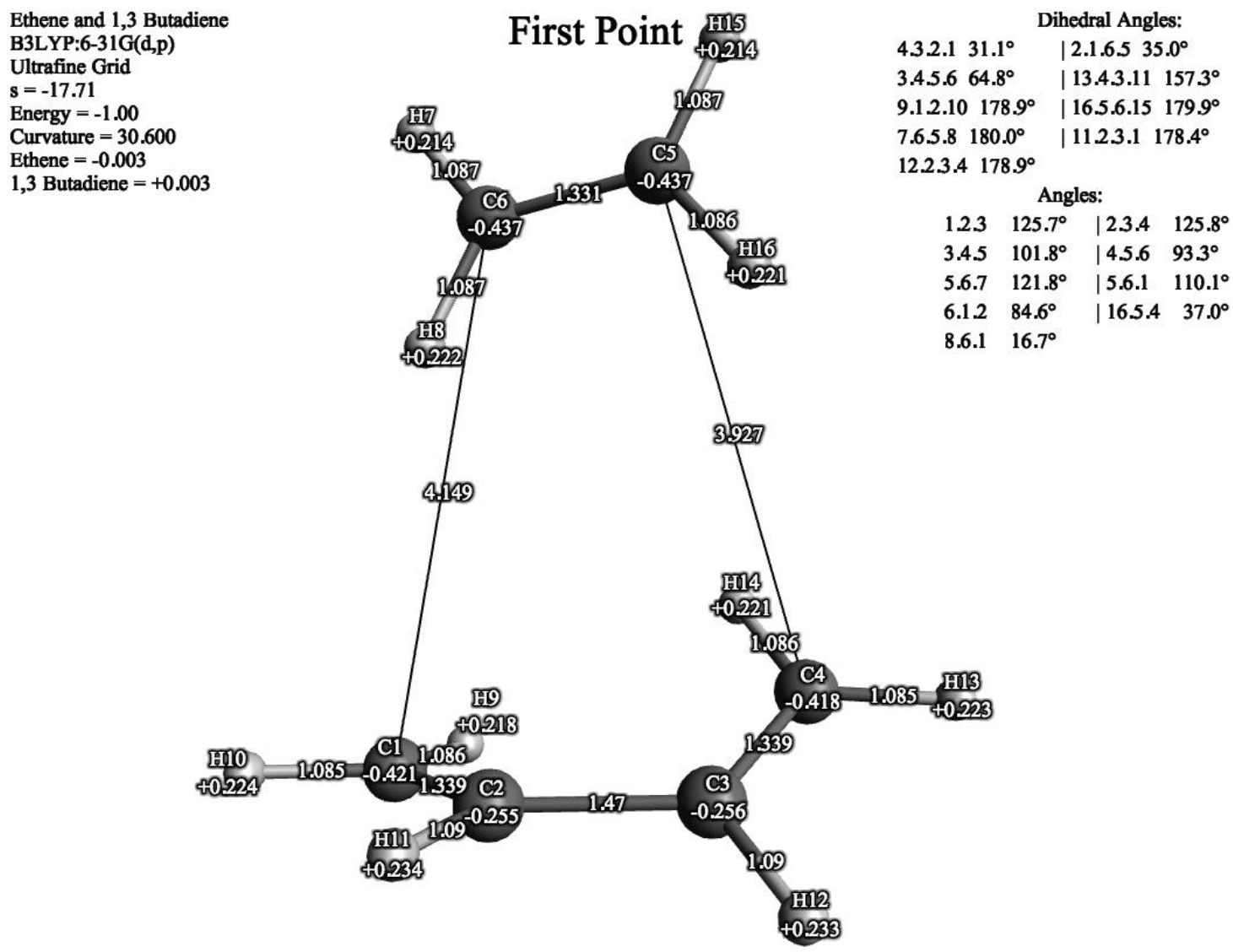

Figure S10: First Point on reaction path. Selected dihedrals, angles, and distances are displayed. Atom symbol, number, and NBO charge are given for each atom. Path length $s$, energy, curvature, and total charge of ethene and 1,3-butadiene molecules are listed. B3LYP/6-31G(d,p) calculation. 


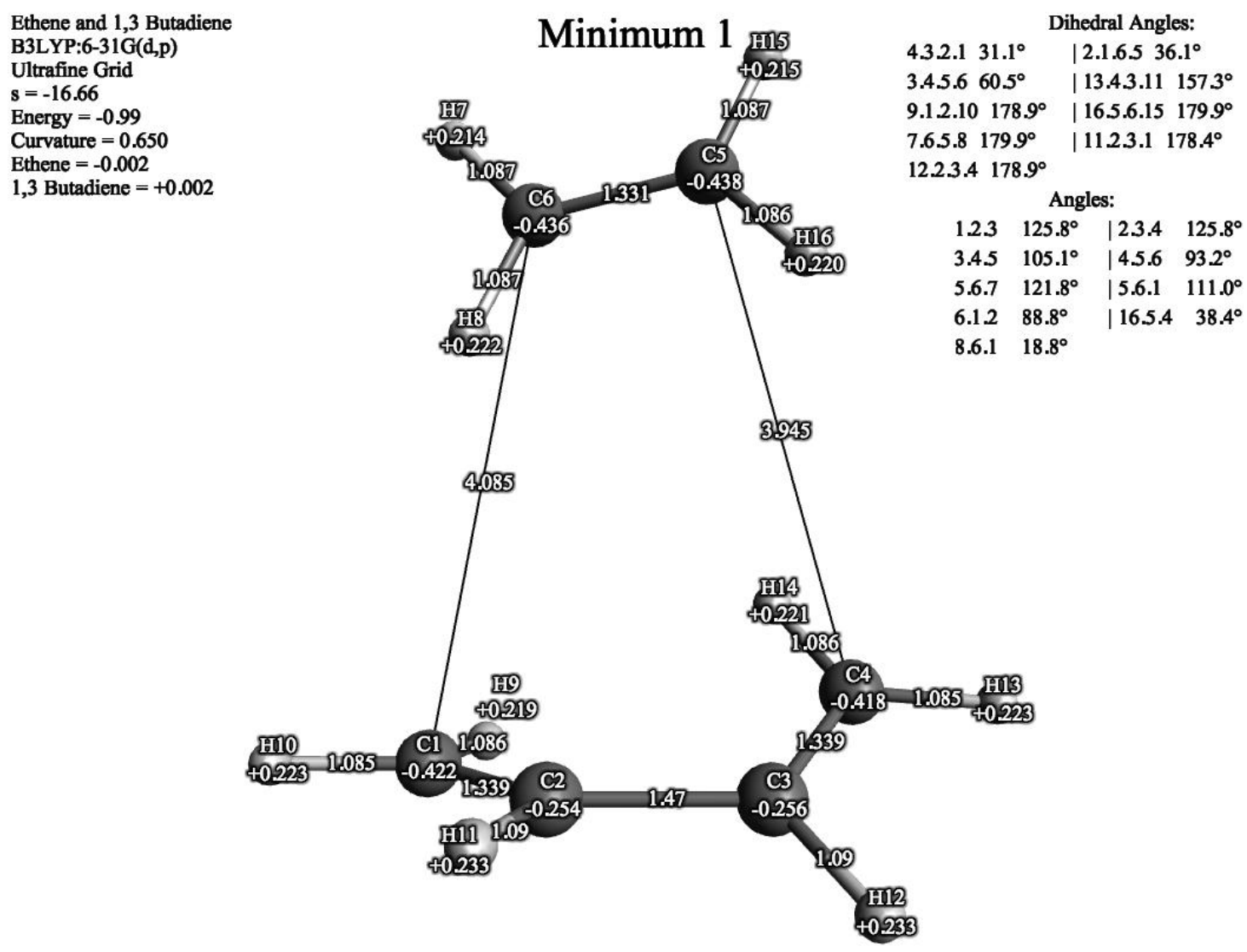

Figure S11: First curvature minimum along reaction path. Selected dihedrals, angles, and distances are displayed. Atom symbol, number, and NBO charge are given for each atom. Path length $s$, energy, curvature, and total charge of ethene and 1,3-butadiene molecules are listed. B3LYP/6$31 \mathrm{G}(\mathrm{d}, \mathrm{p})$ calculation. 


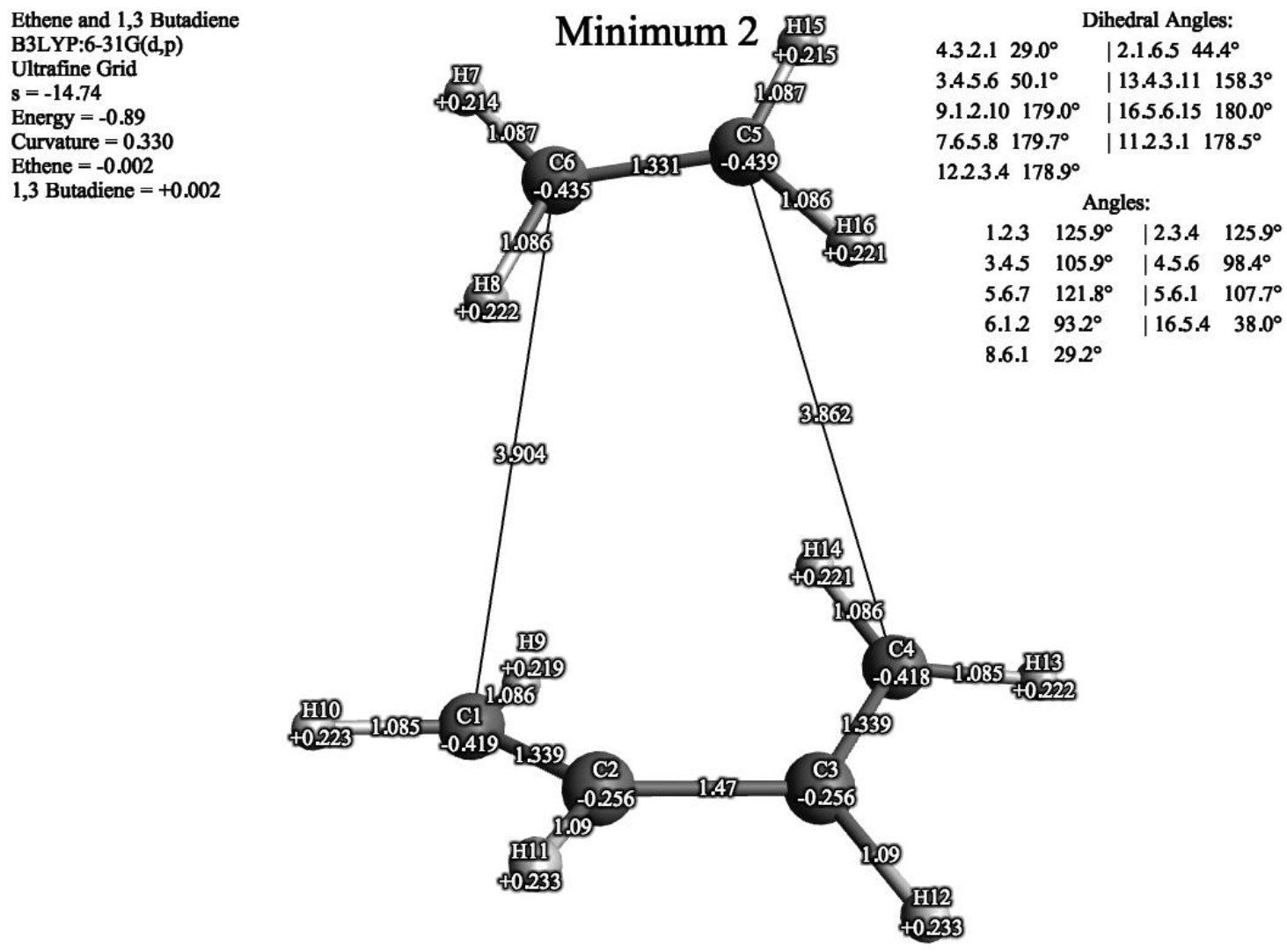

Figure S12: Second curvature minimum along reaction path. Selected dihedrals, angles, and distances are displayed. Atom symbol, number, and NBO charge are given for each atom. Path length $s$, energy, curvature, and total charge of ethene and 1,3-butadiene molecules are listed. B3LYP/6$31 \mathrm{G}(\mathrm{d}, \mathrm{p})$ calculation. 


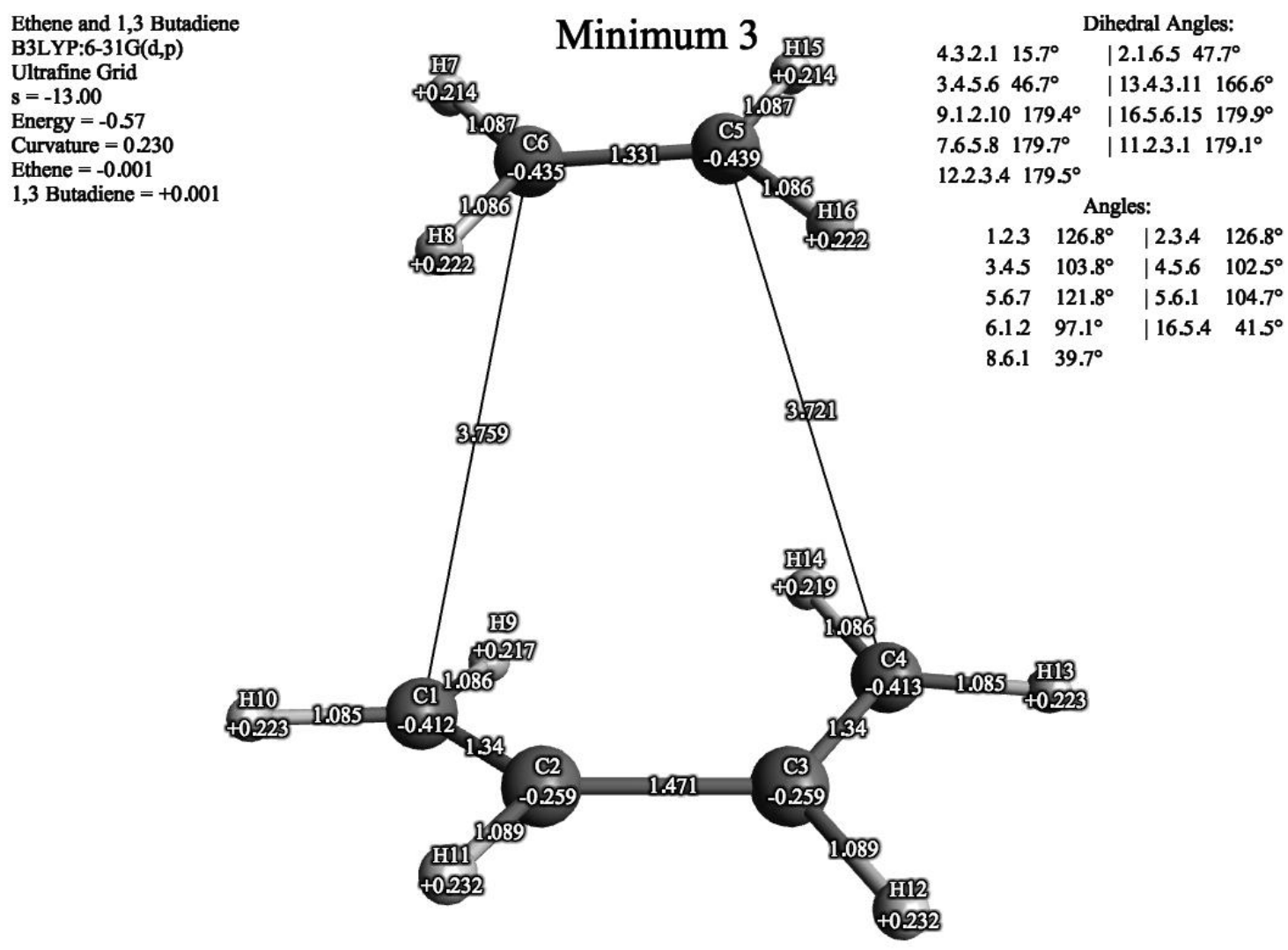

Figure S13: Third curvature minimum along reaction path. Selected dihedrals, angles, and distances are displayed. Atom symbol, number, and NBO charge are given for each atom. Path length $s$, energy, curvature, and total charge of ethene and 1,3-butadiene molecules are listed. B3LYP/6$31 \mathrm{G}(\mathrm{d}, \mathrm{p})$ calculation. 


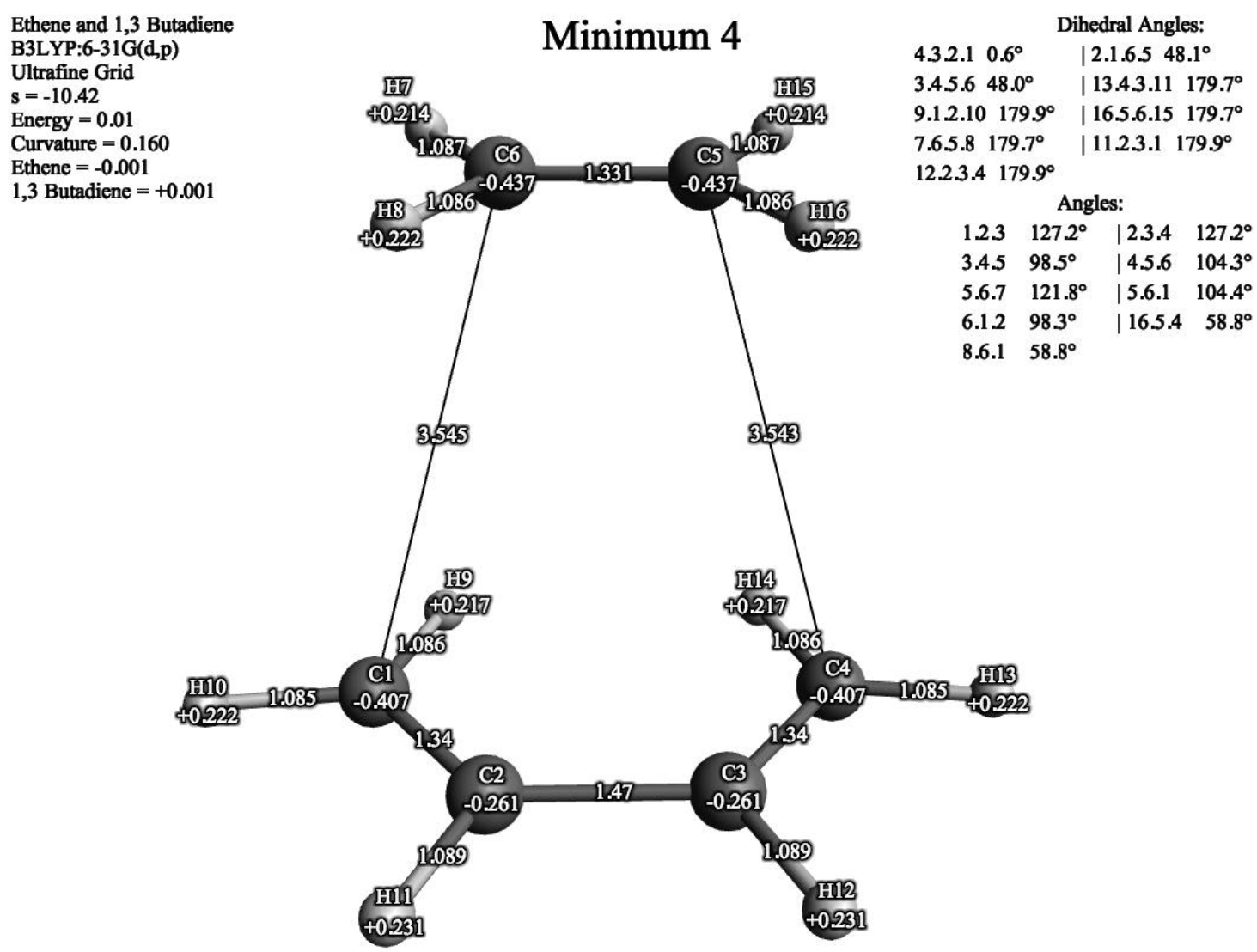

Figure S14: Fourth curvature minimum along reaction path. Selected dihedrals, angles, and distances are displayed. Atom symbol, number, and NBO charge are given for each atom. Path length $s$, energy, curvature, and total charge of ethene and 1,3-butadiene molecules are listed. B3LYP/6$31 \mathrm{G}(\mathrm{d}, \mathrm{p})$ calculation. 


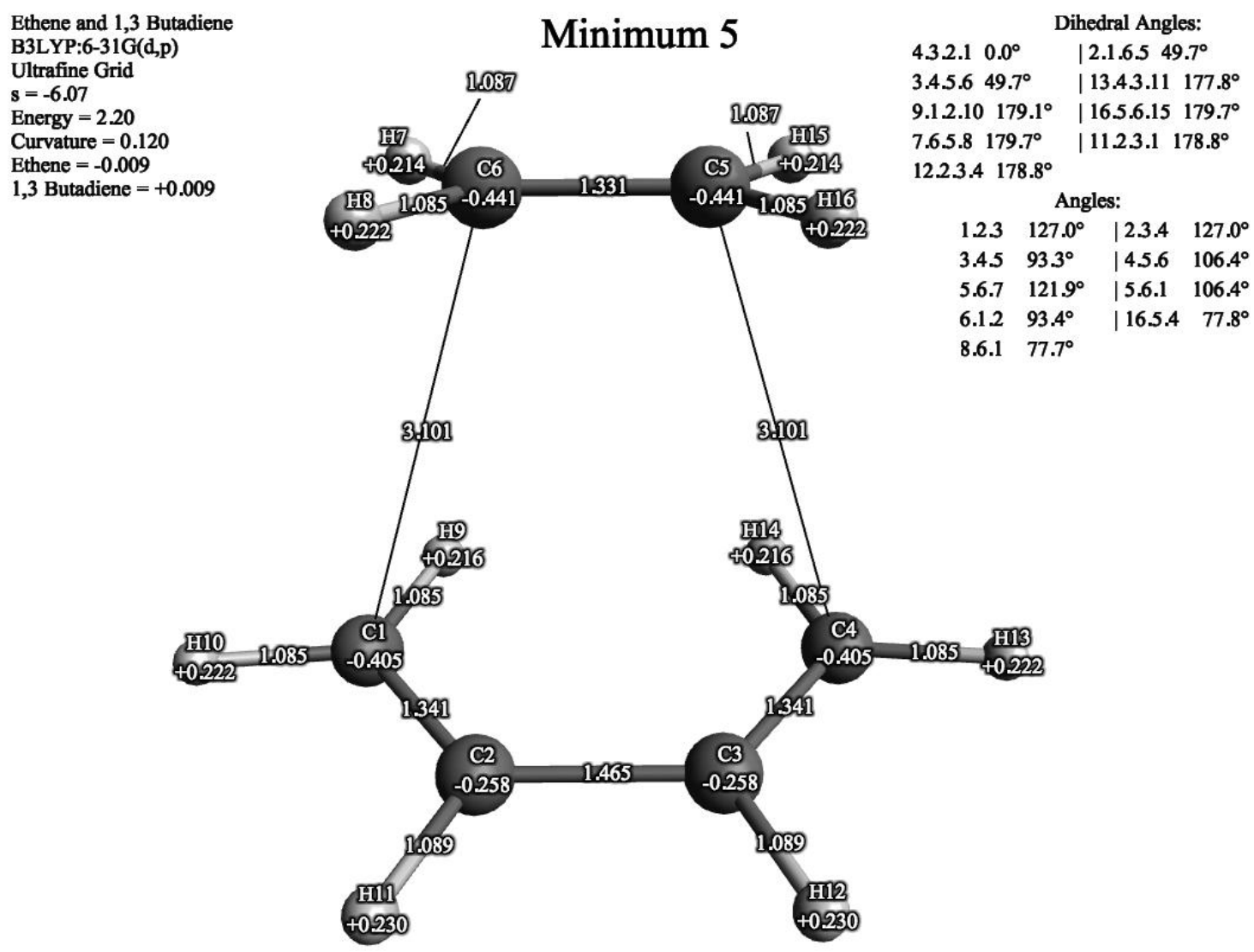

Figure S15: Fifth curvature minimum along reaction path. Selected dihedrals, angles, and distances are displayed. Atom symbol, number, and NBO charge are given for each atom. Path length $s$, energy, curvature, and total charge of ethene and 1,3-butadiene molecules are listed. B3LYP/6$31 \mathrm{G}(\mathrm{d}, \mathrm{p})$ calculation. 


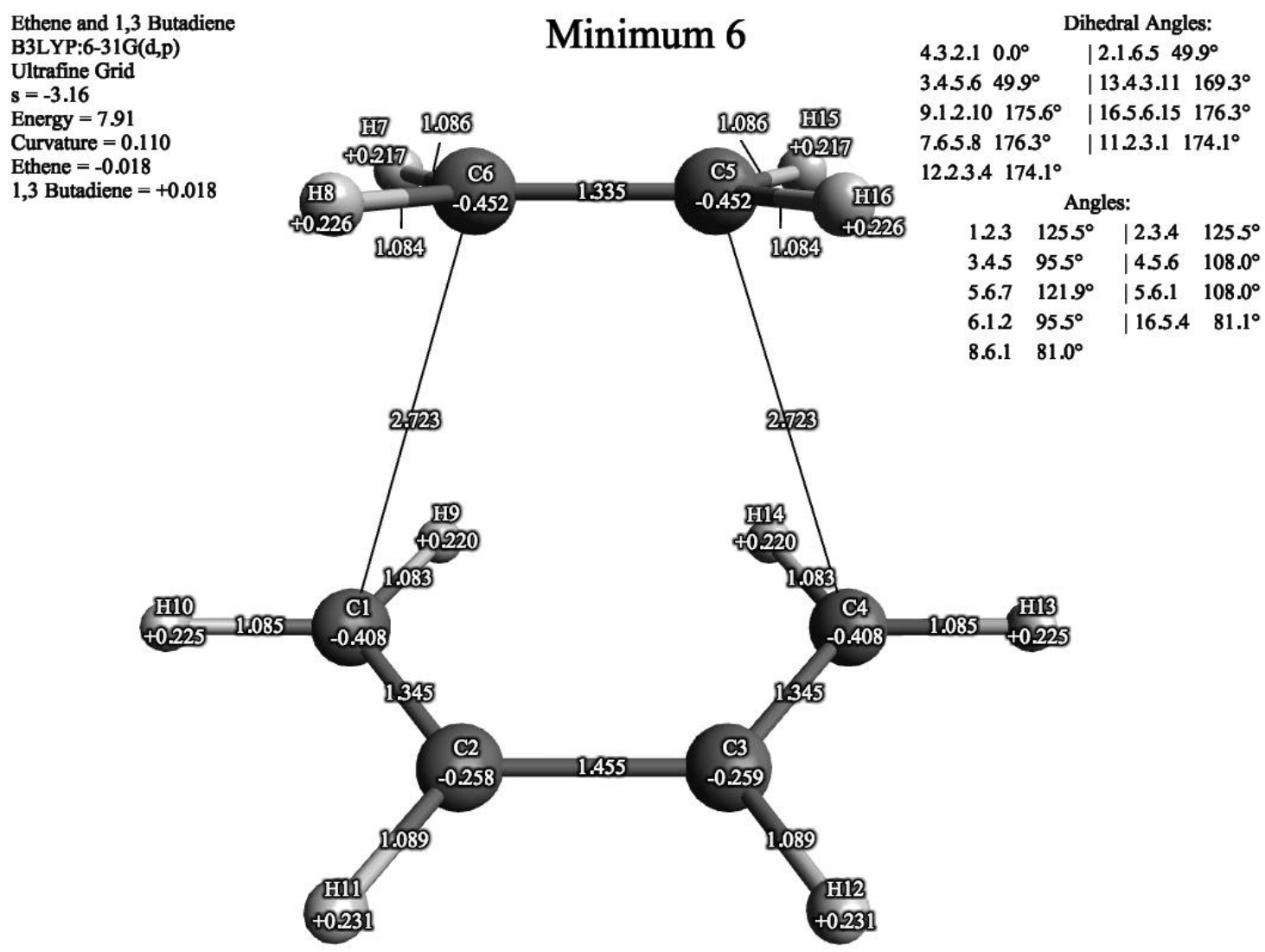

Figure S16: Sixth curvature minimum along reaction path. Selected dihedrals, angles, and distances are displayed. Atom symbol, number, and NBO charge are given for each atom. Path length $s$, energy, curvature, and total charge of ethene and 1,3-butadiene molecules are listed. B3LYP/6$31 \mathrm{G}(\mathrm{d}, \mathrm{p})$ calculation. 


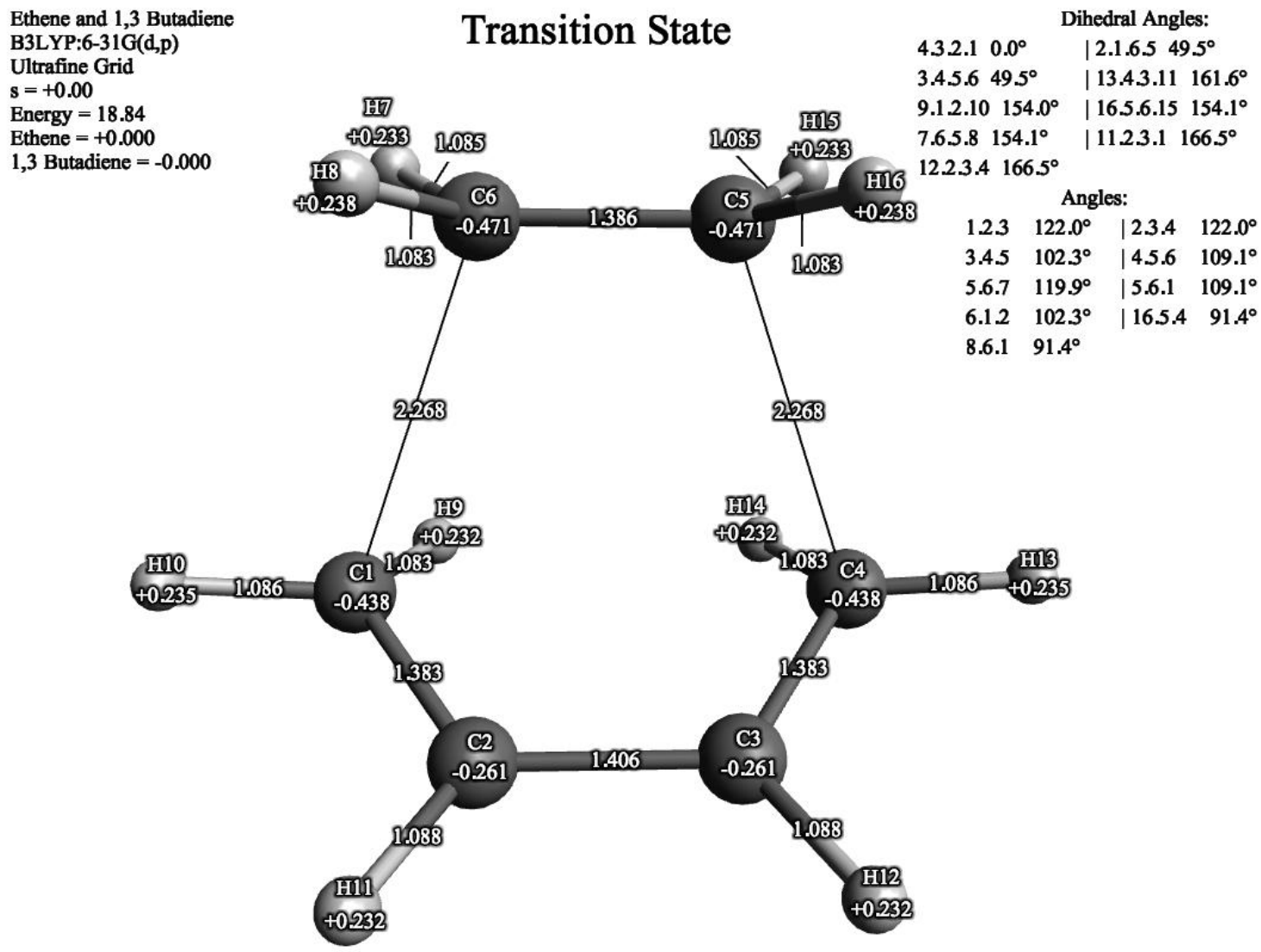

Figure S17: Transition State. Selected dihedrals, angles, and distances are displayed. Atom symbol, number, and NBO charge are given for each atom. Path length $s$, energy, curvature, and total charge of ethene and 1,3-butadiene molecules are listed. B3LYP/6-31G(d,p) calculation. 


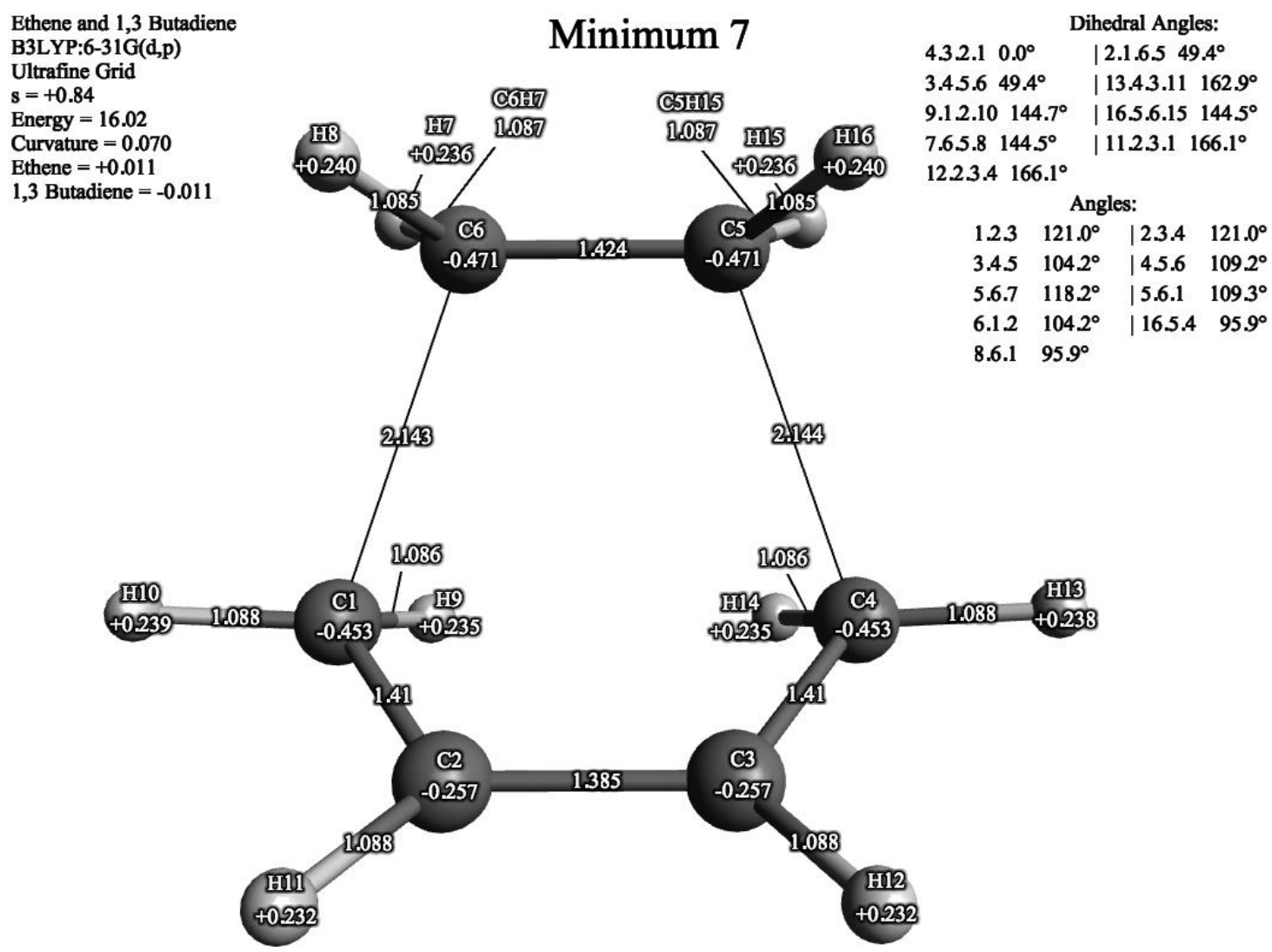

Figure S18: Seventh curvature minimum along reaction path. Selected dihedrals, angles, and distances are displayed. Atom symbol, number, and NBO charge are given for each atom. Path length $s$, energy, curvature, and total charge of ethene and 1,3-butadiene molecules are listed. B3LYP/6-31G $(\mathrm{d}, \mathrm{p})$ calculation. 


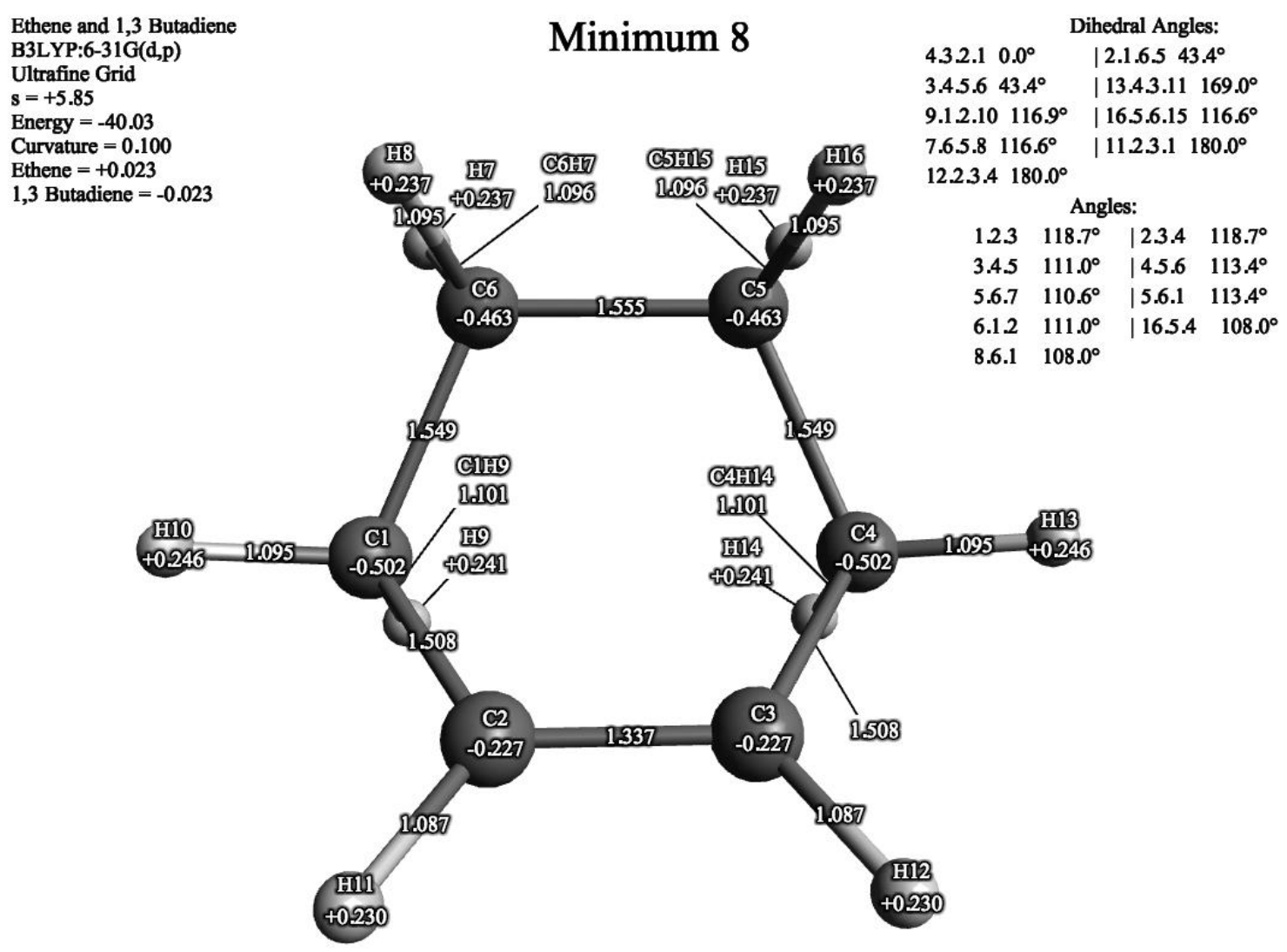

Figure S19: Eighth curvature minimum along reaction path. Selected dihedrals, angles, and distances are displayed. Atom symbol, number, and NBO charge are given for each atom. Path length $s$, energy, curvature, and total charge of ethene and 1,3-butadiene molecules are listed. B3LYP/6$31 \mathrm{G}(\mathrm{d}, \mathrm{p})$ calculation. 


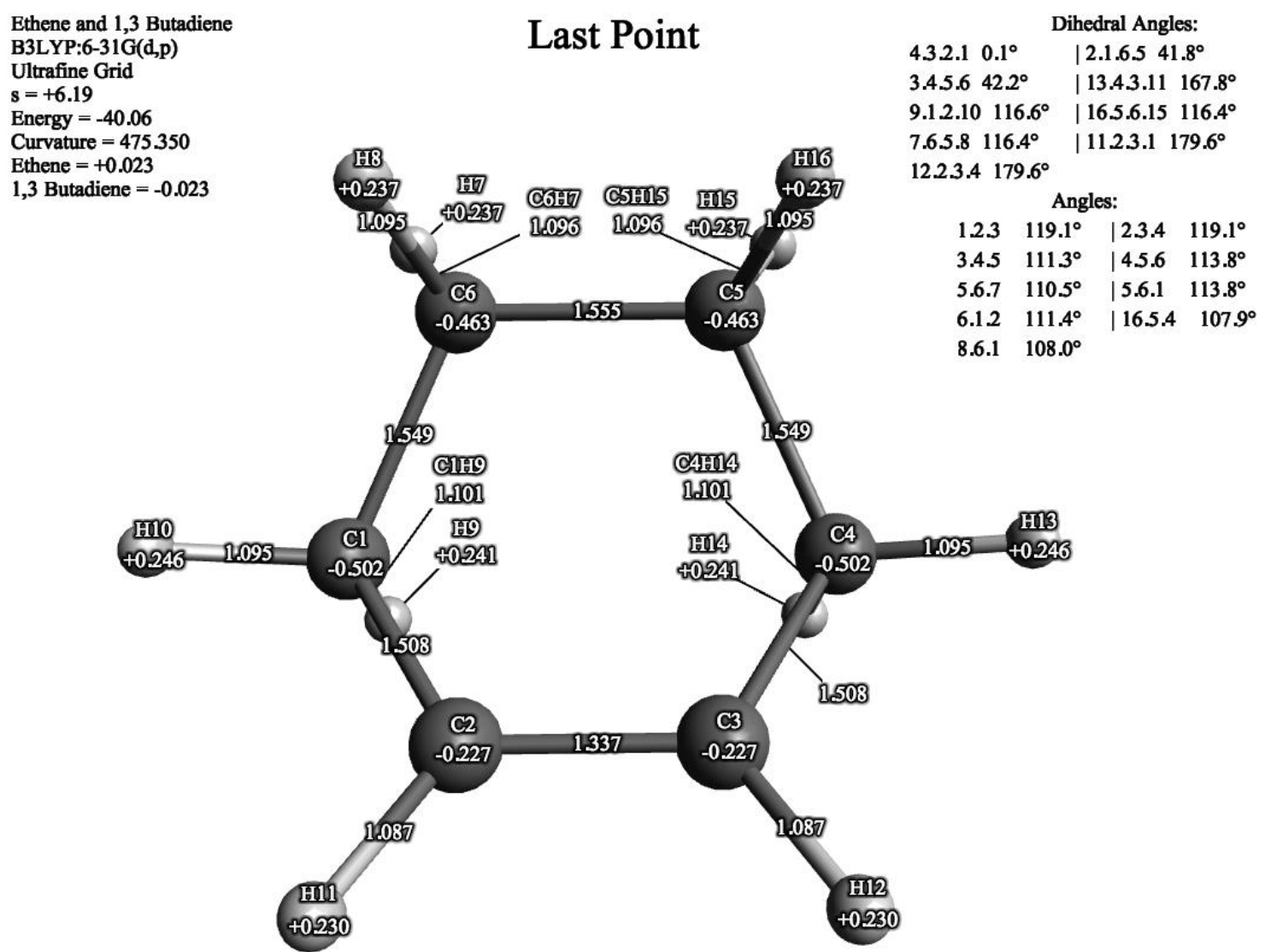

Figure S20: Last Point on reaction path. Selected dihedrals, angles, and distances are displayed. Atom symbol, number, and NBO charge are given for each atom. Path length $s$, energy, curvature, and total charge of ethene and 1,3-butadiene molecules are listed. B3LYP/6-31G(d,p) calculation. 


\section{Cartesian Coordinates of the Reaction Complex at Se- lected Points Along the Reaction Path}

Table S2: Coordinates for First Point ( $\AA$ ) B3LYP/6-31G(d,p)

\begin{tabular}{rrrr} 
Atom & $\mathrm{x}$ & $\mathrm{y}$ & $\mathrm{z}$ \\
\hline $\mathrm{C}$ & -1.50638 & -1.51333 & 0.42052 \\
$\mathrm{C}$ & -1.63606 & -0.59963 & -0.55029 \\
$\mathrm{C}$ & -1.62602 & 0.85867 & -0.3641 \\
$\mathrm{C}$ & -0.94835 & 1.52237 & 0.58122 \\
$\mathrm{C}$ & 2.76036 & 0.52301 & -0.23545 \\
$\mathrm{C}$ & 2.55737 & -0.76101 & 0.04941 \\
$\mathrm{H}$ & 3.36774 & -1.41208 & 0.36725 \\
$\mathrm{H}$ & 1.5709 & -1.21052 & -0.02512 \\
$\mathrm{H}$ & -1.40972 & -1.22762 & 1.46416 \\
$\mathrm{H}$ & -1.51032 & -2.57625 & 0.20093 \\
$\mathrm{H}$ & -1.78868 & -0.94446 & -1.5729 \\
$\mathrm{H}$ & -2.21551 & 1.42958 & -1.08142 \\
$\mathrm{H}$ & -1.01198 & 2.60246 & 0.668 \\
$\mathrm{H}$ & -0.29874 & 1.01061 & 1.28543 \\
$\mathrm{H}$ & 3.74468 & 0.97845 & -0.16329 \\
$\mathrm{H}$ & 1.94612 & 1.1694 & -0.55093
\end{tabular}


Table S3: Coordinates for M1 (̊̊) B3LYP/6-31G(d,p)

\begin{tabular}{rrrr} 
Atom & $\mathrm{x}$ & $\mathrm{y}$ & $\mathrm{z}$ \\
\hline $\mathrm{C}$ & -1.41778 & -1.52948 & 0.4299 \\
$\mathrm{C}$ & -1.66849 & -0.62251 & -0.52335 \\
$\mathrm{C}$ & -1.69983 & 0.83517 & -0.3352 \\
$\mathrm{C}$ & -0.96812 & 1.52624 & 0.54813 \\
$\mathrm{C}$ & 2.7745 & 0.55292 & -0.23129 \\
$\mathrm{C}$ & 2.56873 & -0.73781 & 0.01946 \\
$\mathrm{H}$ & 3.36748 & -1.38764 & 0.36778 \\
$\mathrm{H}$ & 1.59156 & -1.19398 & -0.1136 \\
$\mathrm{H}$ & -1.24096 & -1.23924 & 1.46168 \\
$\mathrm{H}$ & -1.3993 & -2.59196 & 0.20878 \\
$\mathrm{H}$ & -1.8969 & -0.97416 & -1.52942 \\
$\mathrm{H}$ & -2.37239 & 1.38142 & -0.99648 \\
$\mathrm{H}$ & -1.0665 & 2.60312 & 0.64221 \\
$\mathrm{H}$ & -0.23886 & 1.04156 & 1.19089 \\
$\mathrm{H}$ & 3.74941 & 1.01519 & -0.09969 \\
$\mathrm{H}$ & 1.97243 & 1.19851 & -0.57813
\end{tabular}

Table S4: Coordinates for M2 (Å) B3LYP/6-31G(d,p)

\begin{tabular}{rrrr} 
Atom & $\mathrm{x}$ & $\mathrm{y}$ & $\mathrm{z}$ \\
\hline $\mathrm{C}$ & 1.20822 & 1.54528 & 0.48412 \\
$\mathrm{C}$ & 1.62027 & 0.7015 & -0.47112 \\
$\mathrm{C}$ & 1.75887 & -0.75546 & -0.33268 \\
$\mathrm{C}$ & 1.03988 & -1.53878 & 0.48166 \\
$\mathrm{C}$ & -2.65775 & -0.64068 & -0.17918 \\
$\mathrm{C}$ & -2.55842 & 0.68201 & -0.06899 \\
$\mathrm{H}$ & -3.37413 & 1.28682 & 0.3189 \\
$\mathrm{H}$ & -1.65412 & 1.20962 & -0.35816 \\
$\mathrm{H}$ & 0.96101 & 1.20069 & 1.48412 \\
$\mathrm{H}$ & 1.12063 & 2.61092 & 0.2974 \\
$\mathrm{H}$ & 1.90688 & 1.11224 & -1.4391 \\
$\mathrm{H}$ & 2.50948 & -1.21909 & -0.97257 \\
$\mathrm{H}$ & 1.22141 & -2.60731 & 0.54088 \\
$\mathrm{H}$ & 0.24068 & -1.14094 & 1.10025 \\
$\mathrm{H}$ & -3.55838 & -1.17485 & 0.11245 \\
$\mathrm{H}$ & -1.83994 & -1.2413 & -0.56706
\end{tabular}


Table S5: Coordinates for M3 ( $\AA$ ) B3LYP/6-31G(d,p)

\begin{tabular}{rrrr} 
Atom & $\mathrm{x}$ & $\mathrm{y}$ & $\mathrm{z}$ \\
\hline $\mathrm{C}$ & 1.08873 & 1.54531 & 0.50397 \\
$\mathrm{C}$ & 1.62305 & 0.73022 & -0.41559 \\
$\mathrm{C}$ & 1.7077 & -0.73641 & -0.34979 \\
$\mathrm{C}$ & 1.02632 & -1.54341 & 0.47477 \\
$\mathrm{C}$ & -2.53256 & -0.66355 & -0.15961 \\
$\mathrm{C}$ & -2.50995 & 0.66709 & -0.13355 \\
$\mathrm{H}$ & -3.30092 & 1.24404 & 0.33891 \\
$\mathrm{H}$ & -1.69576 & 1.23034 & -0.57959 \\
$\mathrm{H}$ & 0.66461 & 1.173 & 1.43183 \\
$\mathrm{H}$ & 1.06833 & 2.62043 & 0.35726 \\
$\mathrm{H}$ & 2.05913 & 1.17903 & -1.30728 \\
$\mathrm{H}$ & 2.39728 & -1.19144 & -1.0598 \\
$\mathrm{H}$ & 1.17428 & -2.61842 & 0.4556 \\
$\mathrm{H}$ & 0.29572 & -1.16443 & 1.18304 \\
$\mathrm{H}$ & -3.34302 & -1.23192 & 0.28962 \\
$\mathrm{H}$ & -1.73929 & -1.23622 & -0.63089
\end{tabular}

Table S6: Coordinates for M4 (A) B3LYP/6-31G(d,p)

\begin{tabular}{rrrr} 
Atom & $\mathrm{x}$ & $\mathrm{y}$ & $\mathrm{z}$ \\
\hline $\mathrm{C}$ & -0.98998 & -1.54522 & 0.50512 \\
$\mathrm{C}$ & -1.58023 & -0.73489 & -0.38438 \\
$\mathrm{C}$ & -1.58342 & 0.7351 & -0.38214 \\
$\mathrm{C}$ & -0.98757 & 1.54526 & 0.5038 \\
$\mathrm{C}$ & 2.37986 & 0.66545 & -0.16077 \\
$\mathrm{C}$ & 2.37889 & -0.66566 & -0.16025 \\
$\mathrm{H}$ & 2.95957 & -1.23874 & 0.55815 \\
$\mathrm{H}$ & 1.79417 & -1.2359 & -0.87529 \\
$\mathrm{H}$ & -0.42707 & -1.16766 & 1.35309 \\
$\mathrm{H}$ & -1.05393 & -2.62419 & 0.40759 \\
$\mathrm{H}$ & -2.12581 & -1.19348 & -1.20799 \\
$\mathrm{H}$ & -2.13862 & 1.19379 & -1.19923 \\
$\mathrm{H}$ & -1.05805 & 2.62422 & 0.41081 \\
$\mathrm{H}$ & -0.41284 & 1.16752 & 1.34368 \\
$\mathrm{H}$ & 2.96139 & 1.23825 & 0.55716 \\
$\mathrm{H}$ & 1.79597 & 1.23597 & -0.87626
\end{tabular}


Table S7: Coordinates for M5 ( $\AA$ ) B3LYP/6-31G(d,p)

\begin{tabular}{rrrr} 
Atom & $\mathrm{x}$ & $\mathrm{y}$ & $\mathrm{z}$ \\
\hline $\mathrm{C}$ & -0.83966 & -1.54079 & 0.52169 \\
$\mathrm{C}$ & -1.38571 & -0.73254 & -0.39909 \\
$\mathrm{C}$ & -1.38533 & 0.73292 & -0.39914 \\
$\mathrm{C}$ & -0.83924 & 1.54097 & 0.52179 \\
$\mathrm{C}$ & 2.05601 & 0.66542 & -0.16305 \\
$\mathrm{C}$ & 2.05543 & -0.66594 & -0.16303 \\
$\mathrm{H}$ & 2.34146 & -1.24052 & 0.71393 \\
$\mathrm{H}$ & 1.7742 & -1.23856 & -1.04072 \\
$\mathrm{H}$ & -0.34624 & -1.16074 & 1.40982 \\
$\mathrm{H}$ & -0.88465 & -2.62016 & 0.41746 \\
$\mathrm{H}$ & -1.87121 & -1.19389 & -1.258 \\
$\mathrm{H}$ & -1.87026 & 1.19446 & -1.25828 \\
$\mathrm{H}$ & -0.8836 & 2.62035 & 0.41744 \\
$\mathrm{H}$ & -0.34663 & 1.16076 & 1.41029 \\
$\mathrm{H}$ & 2.34253 & 1.23977 & 0.71391 \\
$\mathrm{H}$ & 1.77539 & 1.23829 & -1.04078
\end{tabular}

Table S8: Coordinates for M6 (Å) B3LYP/6-31G(d,p)

\begin{tabular}{rrrr} 
Atom & $\mathrm{x}$ & $\mathrm{y}$ & $\mathrm{z}$ \\
\hline $\mathrm{C}$ & -0.66843 & -1.50781 & 0.51913 \\
$\mathrm{C}$ & -1.32475 & -0.72708 & -0.35783 \\
$\mathrm{C}$ & -1.32456 & 0.72749 & -0.35775 \\
$\mathrm{C}$ & -0.66785 & 1.50795 & 0.51917 \\
$\mathrm{C}$ & 1.82096 & 0.66739 & -0.19624 \\
$\mathrm{C}$ & 1.82061 & -0.66788 & -0.19616 \\
$\mathrm{H}$ & 2.12451 & -1.24199 & 0.67419 \\
$\mathrm{H}$ & 1.57419 & -1.24017 & -1.08301 \\
$\mathrm{H}$ & -0.19083 & -1.10828 & 1.40507 \\
$\mathrm{H}$ & -0.64978 & -2.58749 & 0.41018 \\
$\mathrm{H}$ & -1.82753 & -1.20439 & -1.19735 \\
$\mathrm{H}$ & -1.82741 & 1.20502 & -1.1971 \\
$\mathrm{H}$ & -0.64902 & 2.58764 & 0.41037 \\
$\mathrm{H}$ & -0.19002 & 1.10817 & 1.40487 \\
$\mathrm{H}$ & 2.12517 & 1.24145 & 0.67405 \\
$\mathrm{H}$ & 1.57491 & 1.23972 & -1.08317
\end{tabular}


Table S9: Coordinates for TS $(\AA)$ B3LYP/6-31G(d,p)

\begin{tabular}{rrrr} 
Atom & $\mathrm{x}$ & $\mathrm{y}$ & $\mathrm{z}$ \\
\hline $\mathrm{C}$ & -0.49863 & -0.43431 & 1.436 \\
$\mathrm{C}$ & 0.26106 & -1.32824 & 0.70308 \\
$\mathrm{C}$ & 0.26106 & -1.32824 & -0.70308 \\
$\mathrm{C}$ & -0.49863 & -0.43431 & -1.436 \\
$\mathrm{C}$ & 0.26106 & 1.56899 & -0.69312 \\
$\mathrm{C}$ & 0.26106 & 1.56899 & 0.69312 \\
$\mathrm{H}$ & -0.52468 & 2.08677 & 1.23414 \\
$\mathrm{H}$ & 1.19108 & 1.44666 & 1.23515 \\
$\mathrm{H}$ & -1.45783 & -0.09303 & 1.06599 \\
$\mathrm{H}$ & -0.3871 & -0.38413 & 2.51551 \\
$\mathrm{H}$ & 1.03758 & -1.89495 & 1.21335 \\
$\mathrm{H}$ & 1.03758 & -1.89495 & -1.21335 \\
$\mathrm{H}$ & -0.3871 & -0.38413 & -2.51551 \\
$\mathrm{H}$ & -1.45783 & -0.09303 & -1.06599 \\
$\mathrm{H}$ & -0.52468 & 2.08677 & -1.23414 \\
$\mathrm{H}$ & 1.19108 & 1.44666 & -1.23515
\end{tabular}

Table S10: Coordinates for M7 (A) B3LYP/6-31G(d,p)

\begin{tabular}{rrrr} 
Atom & $\mathrm{x}$ & $\mathrm{y}$ & $\mathrm{z}$ \\
\hline $\mathrm{C}$ & -0.38207 & -1.41865 & 0.4787 \\
$\mathrm{C}$ & -1.32618 & -0.69281 & -0.2755 \\
$\mathrm{C}$ & -1.32677 & 0.69179 & -0.27551 \\
$\mathrm{C}$ & -0.38339 & 1.4186 & 0.47864 \\
$\mathrm{C}$ & 1.51266 & 0.71224 & -0.2304 \\
$\mathrm{C}$ & 1.51293 & -0.71129 & -0.23031 \\
$\mathrm{H}$ & 2.09946 & -1.22433 & 0.52714 \\
$\mathrm{H}$ & 1.47575 & -1.22696 & -1.18411 \\
$\mathrm{H}$ & -0.13404 & -1.07557 & 1.47836 \\
$\mathrm{H}$ & -0.35401 & -2.50102 & 0.37508 \\
$\mathrm{H}$ & -1.90606 & -1.21633 & -1.03313 \\
$\mathrm{H}$ & -1.90718 & 1.21478 & -1.03309 \\
$\mathrm{H}$ & -0.35624 & 2.50097 & 0.37488 \\
$\mathrm{H}$ & -0.13463 & 1.07577 & 1.47819 \\
$\mathrm{H}$ & 2.09874 & 1.22569 & 0.52712 \\
$\mathrm{H}$ & 1.47512 & 1.22787 & -1.18419
\end{tabular}


Table S11: Coordinates for M8 (̊̊) B3LYP/6-31G(d,p)

\begin{tabular}{rrrr} 
Atom & $\mathrm{x}$ & $\mathrm{y}$ & $\mathrm{z}$ \\
\hline $\mathrm{C}$ & -1.39202 & 0.09762 & 0.35533 \\
$\mathrm{C}$ & -0.66388 & 1.30271 & -0.18568 \\
$\mathrm{C}$ & 0.67289 & 1.29809 & -0.18569 \\
$\mathrm{C}$ & 1.39274 & 0.08795 & 0.35523 \\
$\mathrm{C}$ & 0.77312 & -1.21971 & -0.19797 \\
$\mathrm{C}$ & -0.78166 & -1.21426 & -0.19807 \\
$\mathrm{H}$ & -1.16929 & -2.06189 & 0.37785 \\
$\mathrm{H}$ & -1.14163 & -1.34876 & -1.22348 \\
$\mathrm{H}$ & -1.31538 & 0.0908 & 1.4535 \\
$\mathrm{H}$ & -2.462 & 0.13793 & 0.12698 \\
$\mathrm{H}$ & -1.23048 & 2.15011 & -0.56429 \\
$\mathrm{H}$ & 1.24532 & 2.14157 & -0.56431 \\
$\mathrm{H}$ & 2.46292 & 0.1208 & 0.12661 \\
$\mathrm{H}$ & 1.31638 & 0.08181 & 1.45343 \\
$\mathrm{H}$ & 1.15472 & -2.06994 & 0.37814 \\
$\mathrm{H}$ & 1.13226 & -1.35693 & -1.22331
\end{tabular}

Table S12: Coordinates for Last Point $(\AA)$ B3LYP/6-31G(d,p)

\begin{tabular}{rrrr} 
Atom & $\mathrm{x}$ & $\mathrm{y}$ & $\mathrm{z}$ \\
\hline $\mathrm{C}$ & -1.40024 & 0.12023 & 0.34203 \\
$\mathrm{C}$ & -0.6431 & 1.31534 & -0.18074 \\
$\mathrm{C}$ & 0.69327 & 1.28995 & -0.17953 \\
$\mathrm{C}$ & 1.40343 & 0.06631 & 0.3433 \\
$\mathrm{C}$ & 0.75412 & -1.23421 & -0.19175 \\
$\mathrm{C}$ & -0.8006 & -1.20566 & -0.18914 \\
$\mathrm{H}$ & -1.19877 & -2.03928 & 0.3998 \\
$\mathrm{H}$ & -1.16153 & -1.35369 & -1.21236 \\
$\mathrm{H}$ & -1.35512 & 0.11787 & 1.44199 \\
$\mathrm{H}$ & -2.46307 & 0.17691 & 0.08478 \\
$\mathrm{H}$ & -1.1925 & 2.18086 & -0.54351 \\
$\mathrm{H}$ & 1.27582 & 2.13388 & -0.54144 \\
$\mathrm{H}$ & 2.46807 & 0.08264 & 0.08781 \\
$\mathrm{H}$ & 1.3563 & 0.06383 & 1.44318 \\
$\mathrm{H}$ & 1.12332 & -2.08491 & 0.39163 \\
$\mathrm{H}$ & 1.10617 & -1.38989 & -1.21692
\end{tabular}

